\title{
Successful Conversion Surgery for Massive Hepatocellular Carcinoma with Tumor Thrombus in Main Portal Vein after Treatment with Lenvatinib Combined with Toripalimab: A Case Report
}

\section{JingZhong OuYang ( $\sim 15857701637 @ 163 . c o m)$}

Affiliated Tumor Hospital of Zhengzhou University: Henan Cancer Hospital https://orcid.org/00000002-3398-6465

Yanzhao Zhou

Affiliated Tumor Hospital of Zhengzhou University: Henan Cancer Hospital

Zhengzheng Wang

Affiliated Tumor Hospital of Zhengzhou University: Henan Cancer Hospital

Qingjun Li

Affiliated Tumor Hospital of Zhengzhou University: Henan Cancer Hospital

Jinxue Zhou

Affiliated Tumor Hospital of Zhengzhou University: Henan Cancer Hospital

\section{Research Article}

Keywords: Hepatocellular carcinoma, Portal vein invasion, Lenvatinib, Toripalimab, Conversion therapy, CR

Posted Date: September 2nd, 2021

DOl: https://doi.org/10.21203/rs.3.rs-840640/v1

License: (c) (i) This work is licensed under a Creative Commons Attribution 4.0 International License. Read Full License 


\section{Abstract}

\section{Background:}

Advanced hepatocellular carcinoma (HCC) with Portal vein invasion has an extremely dismal prognosis. We report a rare case of advanced HCC with portal vein tumor thrombus (PVTT). First, Lenvatinib(Len) combined with Toripalimab(Tor) was treated. The patient was successfully treated with radical surgical resection after the tumor thrombus shrank. Postoperative pathology showed complete response (CR).

\section{Case presentation:}

A 52-year-old male patient had a massive liver cancer in his right liver, and the tumor thrombus grew to the main portal vein. He passed 4 cycles of Len combined with Tor, the tumor shrank rapidly, the level of tumor markers dropped rapidly, The tumor thrombus was successfully confined from the main portal vein to the right branch of the portal vein. Therefore, the patient underwent a right hepatectomy and successfully removed a complete PVTT. Histopathological results showed that the primary tumor and tumor thrombus were only infiltrated by inflammatory cells, and there were no viable tumor cells.

\section{Conclusions:}

Len combined with Tor can be used as a preoperative neoadjuvant regimen for the treatment of advanced $\mathrm{HCC}$ with massive macrovascular invasion.

\section{Background}

In recent years, the treatment of HCC has formed a multi-method and multi-disciplinary comprehensive treatment model, such as local treatment and systemic treatment, because $70 \%$ of HCC patients are advanced when they are discovered, a single treatment method cannot satisfy the treatment needs[1].Median overall survival(mOS) and median time to progression(mTTP) of patients with advanced HCC treated with Sorafenib are about 3 months longer than the control group, but the objective response rate(ORR) is only $2 \%$ (RECIST).Len only has ORR of $18.8 \%$,whose first-line treatment effect is not inferior to Sorafenib, (RECIST1.1)[2, 3]. By blocking programmed cell death-1(PD-1) and programmed cell death ligand-1(PD-L1), immune checkpoint inhibitors(ICls) can reduce the apoptosis of T lymphocytes and improve the immune microenvironment of tumors(TIM), thus inducing $\mathrm{T}$ lymphocytes to resume their anti-tumor effect. There are Nivolumab, Pembrolizumab, Camrelizumab, etc[4].Tor is a recombinant humanized PD-1 monoclonal antibody. It binds to PD-1 and prevents PD-1 from binding to PD-L1 and PD$L 2$, thus exerting its anti-tumor effect. It has a good effect in malignant tumors such as melanoma $[5,6]$. Hypoxia and acidosis of TIM caused by abnormal blood vessels in tumors leads to the up-regulation of PD-L1 expression on tumor-associated macrophages, dendritic cells, tumor cells and PD-1 expression on inhibitory cytotoxic T lymphocytes through VEGF, ANG2 and other cytokines. PD-1-PD-L1 pathway is activated. The use of anti-VEGF molecular targeted drugs (MTDs) to improve TIM can play a synergistic anti-cancer effect with ICls [7]. Studies have shown that the ORR of Len and Tor combined with hepatic 
artery infusion chemotherapy(HAIC) in the treatment of advanced $\mathrm{HCC}$ is as high as $48 \%$, of which the complete response rate(CRR) is $14.1 \%$, and the CRR of intrahepatic target lesions is $21.1 \%$. It shows a good application prospect in conversion therapy [8]. Here is a report of a newly diagnosed patient with massive HCC and PVTT III. After Len combined with Tor treatment, RO resection was successfully achieved.

\section{Case Presentation}

On February 12, 2021, a 52-year-old male weighing $76 \mathrm{~kg}$ was found with liver space occupying lesions in a local hospital. Conventional computed tomography (CT) and enhanced magnetic resonance imaging (MRI) showed right lobe mass of liver. HCC was considered in the imaging department of our hospital. On February 19, 2021, On February 19, 2021, he was transferred to our hospital with " epigastric discomfort for more than 3 weeks". The patient had no special discomfort during the course of the disease. The patient has a history of untreated viral hepatitis B for 20 years and has not received any physical examination in the past 5 years. Physical examination: no special signs. Laboratory examination: total bilirubin(TBil) 16.9umol/L (normal:0-26), direct bilirubin(DBil) 8.1 umol/L (normal:0-8), serum albumin level (Alb) 39.4g/L (normal:40-55),glutamic pyruvic transaminase(GPT)47u/L (normal:9-50), glutamic oxaloacetic transaminase(GOT) 45u/L (normal:15-40), alkaline phosphatase(ALP) 211u/L (normal:45125). Prothrombin time(PT) $12.1 \mathrm{~s}$ (normal:10-14), HBsAg (+), HBeAg (+), HBcAb (+), the quantitation of HBV DNA: $2.36 * 10^{3} \mathrm{IU} / \mathrm{ml}$, Alpha-fetoprotein(AFP):> $1210 \mathrm{ng} / \mathrm{mL}$ (normal: $0-7$ ). Enhanced CT of the upper abdomen revealed: there was a low-density mass in the right lobe of the liver, the largest crosssection is $135 \mathrm{~mm} \times 104 \mathrm{~mm}$ (Fig. 1a); the mass is unevenly enhanced in the arterial phase, and the main portal vein and right branch have low-density filling defects (Fig. 1b).

After multidisciplinary comprehensive diagnosis and treatment, it was considered that the patient had the indication of radical resection, and other local or systemic treatments were recommended to alleviate the progress of the disease. We informed the patients and his families about his condition and treatment plans, and finally decided to use MTDs combined with ICls treatment. On February 21, 2021, the patient was given "Tor" $240 \mathrm{mg}$ i.v. combined with "Len" $8 \mathrm{mg}$ p.o. qd for the first time, and the patient was given Entecavir Tablets $0.5 \mathrm{mg}$ p.o. qd antiviral therapy. Later, the patients were treated with the same regimen on March 12, 2021, April 02, 2021 and April 22, 2021 respectively. The changes of AFP during the treatment process are shown in Fig. 2, and no adverse events occurred during the treatment process. The enhanced CT of the upper abdomen on April 22, 2021 showed: the mass of the right lobe of the liver was reduced compared to before, with a maximum cross-section of $109 \mathrm{~mm} \times 85 \mathrm{~mm}$ (Fig. 1c); the low-density filling defect in the portal vein was narrowed down and confined to the right branch of the portal vein (Fig. 1d). 
Table 1

3D reconstruction data and calculation of future liver volume ratio

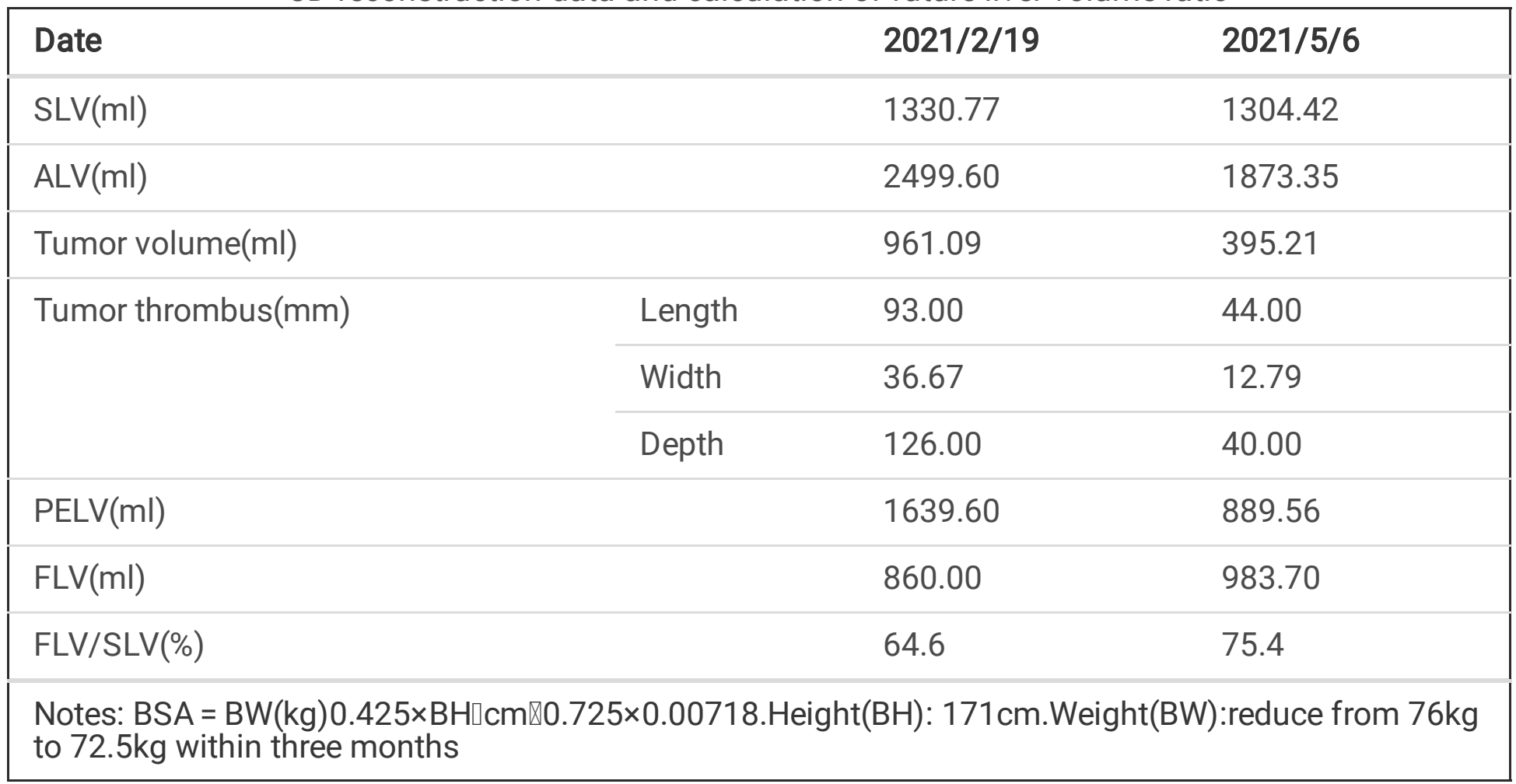

Abbreviations: SLV, standard liver volume; ALV, actual liver volume; PELV, predicted excisional liver volume; FLV, future liver volume; SLV = 706.2×BSA(m2) + 2.4.

May 6, 2021, the patient came to the hospital for review. Laboratory examination: TBil 12.8umol/L, DBil 6.1umol/L, Alb 41.1g/L, GPT 25u/L, GOT 28u/L,ALP 198u/L.PT 12.16s,the quantitation of HBV DNA< $1 * 102 \mathrm{IU} / \mathrm{ml}$, AFP $2.45 \mathrm{ng} / \mathrm{ml}$, indocyanine green retention rate at 15 minute(ICG-R15)18.6\%.Enhanced MRI of the upper abdomen showed that a mass in the right lobe of the liver, mixed with slightly high signal on T1 and T2, and a widening of the right branch of the portal vein with slightly high signal on T2 (Fig. 1e). The three-dimensional visualization results based on the CT images of February 19, 2021 and the MRI images of May 6, 2021 were obtained by using the mixed reality operation planning simulation system software, and the right hepatectomy was simulated, and the postoperative remaining liver volume was calculated (Fig. 3a, b, c, d, Table 1). Patient and his families strongly requested surgical treatment, We fully informed the risks and possible prognosis of the surgery, and signed a written informed consent form. On May 12, 2021, "right hepatectomy plus cholecystectomy plus portal vein tumor thrombus removal and intestinal adhesion lysis" was performed under general anesthesia. Mild liver cirrhosis was found during the operation, and the tumor was confined to segments V, VI, VII, and VIII of the liver, with a size of approximately $140 \mathrm{~mm} \times 100 \mathrm{~mm}$. The dissection of the tumor showed that the size of the tumor was $90 \mathrm{~mm} \times 80 \mathrm{~mm} \times 85 \mathrm{~mm}$, grayish yellow and grayish red, medium texture, with a large amount of necrotic tissue in the middle, and intact tumor thrombus could be seen in the portal vein (Fig. 4a). The postoperative pathology report showed: (right hepatic)massive necrosis, fibrous tissue hyperplasia, inflammatory cell infiltration and hemosiderin under microscope(Fig. 4b); (tumor thrombus) degenerative necrotic material and a large amount of inflammatory cell infiltration in a few of fibrous connective tissue (Fig. 4c);(the margin of hepatectomy ) no malignant indication was seen. The patient was given 
symptomatic treatment such as antibiotics and liver protection after operation, and he was discharged safely 9 days after operation with no complication. 
Table 2

Reported in the literature of HCC to surgery after MTD/ICI treatment

\begin{tabular}{|c|c|c|c|c|c|c|}
\hline Drugs & Citation & Number & Regimen & Pathology & $\begin{array}{l}\text { Follow- } \\
\text { up time } \\
\text { (months) }\end{array}$ & Prognosis \\
\hline Sorafenib & $\begin{array}{l}\text { Clin J } \\
\text { Gastroenterol.2015 } \\
\text { Oct ;8(5):300 }\end{array}$ & 1 & EBRT & $\mathrm{pCR}$ & 9 & DF \\
\hline \multirow[t]{2}{*}{ Sorafenib } & \multirow{2}{*}{$\begin{array}{l}\text { Int Surg } \\
2015 ; 100: 908\end{array}$} & \multirow[t]{2}{*}{1} & TACE+ & \multirow[t]{2}{*}{-} & \multirow[t]{2}{*}{48} & \multirow[t]{2}{*}{ DF } \\
\hline & & & 3-DCRT & & & \\
\hline \multirow[t]{2}{*}{ Sorafenib } & \multirow{2}{*}{$\begin{array}{l}\text { Liver Int.2011 May } \\
; 31(5): 740\end{array}$} & \multirow[t]{2}{*}{2} & - & $\mathrm{pCR}$ & 12 & DF \\
\hline & & & - & $\mathrm{pCR}$ & 16 & DF \\
\hline Sorafenib & $\begin{array}{l}\text { Per Med.2020 } 03 \\
; 17(2): 83\end{array}$ & 1 & -- & $\mathrm{pCR}$ & 12 & DF \\
\hline \multirow[t]{2}{*}{ Sorafenib } & \multirow{2}{*}{$\begin{array}{l}\text { Gan To Kagaku } \\
\text { Ryoho. } 2015 \text { Nov } \\
; 42(12): 1638\end{array}$} & \multirow[t]{2}{*}{1} & TAE+ & \multirow[t]{2}{*}{-} & \multirow[t]{2}{*}{10} & \multirow[t]{2}{*}{ DF } \\
\hline & & & TACE & & & \\
\hline \multirow[t]{2}{*}{ Sorafenib } & \multirow{2}{*}{$\begin{array}{l}\text { Gan To Kagaku } \\
\text { Ryoho. } 2020 \text { Mar } \\
; 47(3): 502\end{array}$} & \multirow[t]{2}{*}{1} & TAE+ & \multirow[t]{2}{*}{-} & \multirow[t]{2}{*}{18} & \multirow[t]{2}{*}{ DF } \\
\hline & & & TACE & & & \\
\hline \multirow[t]{2}{*}{ Sorafenib } & \multirow{2}{*}{$\begin{array}{l}\text { Int J Hepatol.2011 } \\
; 2011: 791013\end{array}$} & \multirow[t]{2}{*}{2} & - & pPR & 12 & recur \\
\hline & & & - & pPR & 6 & DF \\
\hline \multirow[t]{3}{*}{ Len } & \multirow{3}{*}{$\begin{array}{l}\text { Medicine (2020) } \\
99: 42\end{array}$} & \multirow[t]{3}{*}{3} & TAE & $\mathrm{pCR}$ & 6 & DF \\
\hline & & & -- & $\mathrm{pCR}$ & 3 & DF \\
\hline & & & - & $\mathrm{pCR}$ & 6 & DF \\
\hline Len & $\begin{array}{l}\text { World J Hepatol } \\
\text { 2021 March 27; } \\
\text { 13(3): } 384\end{array}$ & 1 & - & pPR & 8 & DF \\
\hline Len & $\begin{array}{l}\text { Anticancer } \\
\text { Res.2019 Oct } \\
; 39(10): 5695\end{array}$ & 1 & TACE & pPR & 12 & DF \\
\hline Len & $\begin{array}{l}\text { Liver Cancer } \\
\text { 2020;9:358 }\end{array}$ & 1 & -- & $\mathrm{pCR}$ & 5 & DF \\
\hline Len & $\begin{array}{l}\text { World J Hepatol } \\
\text { 2020 December 27; } \\
\text { 12(12): } 1349\end{array}$ & 1 & -- & pPR & 12 & DF \\
\hline
\end{tabular}

Abbreviations: 3-DCRT,3-dimensional conformal radiation therapy; TAE, Hepatic artery embolization; pCR, pathological complete response; pPR, pathological partial response; DF, Disease free. 


\begin{tabular}{|c|c|c|c|c|c|c|}
\hline Drugs & Citation & Number & Regimen & Pathology & $\begin{array}{l}\text { Follow- } \\
\text { up time } \\
\text { (months) }\end{array}$ & Prognosis \\
\hline Len & $\begin{array}{l}\text { Surg Case Rep.2020 } \\
\text { Dec } 10 ; 6(1): 318\end{array}$ & 1 & - & pPR & 12 & DF \\
\hline \multirow[t]{2}{*}{ Len } & \multirow{2}{*}{$\begin{array}{l}\text { Hepatol Res.2021 } \\
\text { May } 13\end{array}$} & \multirow[t]{2}{*}{2} & TACE & $\mathrm{pCR}$ & - & - \\
\hline & & & TACE & pPR & - & - \\
\hline Len & $\begin{array}{l}\text { Ann Surg } \\
\text { Oncol.2021 Apr } 26\end{array}$ & 16 & - & RO: 9 & mOS: 19 & - \\
\hline Regorafenib & $\begin{array}{l}\text { Clinical Journal of } \\
\text { Gastroenterology } \\
\text { (2020) 13:428 }\end{array}$ & 1 & -- & pPR & 8 & DF \\
\hline $\begin{array}{l}\text { Len+ } \\
\text { Nivolum }\end{array}$ & $\begin{array}{l}\text { OncoT argets and } \\
\text { Therapy 2019:12 } \\
7355\end{array}$ & 1 & -- & $\mathrm{pCR}$ & - & - \\
\hline $\begin{array}{l}\text { Cabozantinib+ } \\
\text { Nivolumab }\end{array}$ & $\begin{array}{l}\text { J Immunother } \\
\text { Cancer } \\
\text { 2020;8:e000932 }\end{array}$ & 1 & -- & pPR & 24 & DF \\
\hline
\end{tabular}

\section{Discussion}

In a narrow sense, conversion therapy refers to tumors that cannot be removed by primary surgery achieve the effect of tumor reduction and downstage through preoperative treatment, so as to achieve the purpose of radical resection at the surgical technical level. However, the hepatectomy is often limited by requirements such as liver function and FLV, so the author thinks, conversion therapy of HCC is not limited to the decline of preoperative clinical stage, but it should be through multidisciplinary comprehensive diagnosis and treatment, using personalized plans to make the disease develop in a direction that is more conducive to the prognosis[9].The conversion therapy of HCC is not a new concept of treatment, Sitzmann et al. treated unresectable advanced HCC by external radiotherapy combined with $1^{131}$-antiferritin chemotherapy in 1993 , the tumor reduction rate of 14 patients was more than $50 \%$ and hepatectomy was performed successfully[10]. There are various specific conversion treatment strategies for HCC, but the response rate of both local treatment and systemic treatment is low, and it cannot achieve the ORR as high as $70 \%$ like colorectal cancer liver metastasis, which limits the development of conversion treatment for HCC [11,12]. HCC is the fourth cause of cancer-related death in the world, and the five-year survival rate of patients with advanced HCC treated by systematic treatment is less than $20 \%$. Therefore, while perfecting the monitoring system for high-risk patients with liver cancer, 
transforming advanced HCC that cannot be removed by primary surgery into operable, is of great significance for improving the prognosis and the OS of HCC patients $[13,14]$.

Due to the anatomical characteristics of the liver and the biological characteristics of HCC, HCC easily invades the intrahepatic vascular system, especially the formation of PVTT. It has been reported that the proportion of advanced patients with PVTT in the first diagnosis of HCC is $10-40 \%$. Compared with the advanced patients without PVTT, the OS decreased from 10-24 months to 2- 4 months $[15,16]$. VP classification of Japan [17] and Cheng's classification of China [18] are commonly used clinically to classify PVTT. The main difference between the two classifications are that Vp4 includes PVTT $₫$ which invades the main portal vein and PVTT $₫$ which invades the superior mesenteric vein, while PVTT 『includes VP1 and VP2 that tumor thrombus is confined to the secondary portal vein and below. According to EASL guidelines and NCCN guidelines, stage Barcelona clinic liver cancer(BCLC)C $\mathrm{HCC}$ with portal vein invasion is an absolute contraindication for surgical resection, and systematic treatment is recommended[19,20]. However, the CNLC guidelines believe that for tumors confined to the hemi-liver and PVTT confined to the left or right branches, that is, PVTT囚and PVTT区(Vp0, 1, 2, 3), surgical resection of tumor and removal of thrombus through portal vein should be considered if the technology permits. And hepatic arterial chemoembolization(TACE), portal vein chemotherapy or other systematic treatment can be given after operation[21]. Studies have shown that surgical resection can still provide better survival benefits than TACE and other non-operative treatments for patients with advanced HCC and PVTT, especially for patients with single mass and PVTT $\nabla$ and $\otimes[22,23]$.Therefore, neoadjuvant therapy is given before surgery to successfully reduce the tumor and improve the stage, or improve the classification of PVTT in patients with advanced HCC, and then radical resection of the tumor is given. That is significant for advanced patients who do not meet the surgical resection standards specified in the guidelines. The preoperative imaging of our case showed PVTT \. After 4 cycles of MTDs and ICls combined therapy, not only the mass was significantly reduced (58.9\% smaller in size), but also the PVTT

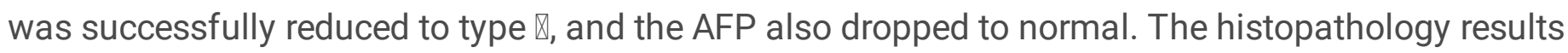
showed $\mathrm{CR}$, indicating a good prognosis of the patient.

The conversion therapy of HCC has always been limited by the low response rate of adjuvant therapy. In recent years, MTDs and ICls have been proven to be effective in the treatment of advanced HCC, However, the objective response rate of monotherapy is still only $2 \%-24.1 \%[2,3,24,25,26]$. In 2020 , several clinical studies have proved that the combination of the two drugs shows a 1: $1>2$ effect in advanced HCC, which brings a new approach for the conversion therapy of HCC. The phase III clinical study IMbrave-150 showed that although the ORR of Bevacizumab combined with Atezolizumab in the treatment of advanced HCC was only $27.3 \%$, the CRR was as high as $5.5 \%$, while none of the patients in Sorafenib group achieved CR [27]. The KEYNOTE-524 clinical study enrolled 104 patients with advanced HCC. The ORR of Len combined with Pembrolizumab was as high as $46 \%$, of which the CRR was $11 \%$ and the partial response rate (PRR) was 35\% [28]. The PRR of Apatinib combined with Camrelizumab in the treatment of stage BCLC C HCC also reached $50 \%$ [29]. In the study of combined therapy with double ICls, the ORR of $22.7 \%-31 \%$ was also achieved, which is twice or higher than that of single drug [30,31]. The ORR of the IMbrave-150 study was $27.3 \%$, of which the disease-free progression rate in 6 months was 
$54.5 \%$. The KEYNOTE-524 clinical study showed that $73 \%$ of the $46 \%$ objective response patients achieved long-term remission for 6 months or more, indicating that the combination of MTDs and ICls can improve the TIM in the anti-tumor process and play a long-term role in the anti-tumor effect.

The conversional therapies of HCC are various, including TACE, HAIC, stereotactic body radiation therapy (SBRT), concurrent radiochemotherapy (CCRT), transarterial radioembolization (TARE), MTDs, ICls and the combined application of various treatments. There are some retrospective analyses and case reports, but is still a lack of prospective studies to prove the efficacy of specific conversional treatments for HCC $[32,33,34,35]$. With the development of MTDs and ICls, successful cases of the two drugs in the conversional therapy of HCC are often reported. Many patients not only have OR or CR pathologically after treatment, but also achieve a tumor-free survival of up to 48 months in the later follow-up (Table 2). There are few research reports on MTDs combined ICls for conversional therapy at present. Chen et al. reported a case of massive HCC that was intolerant by Sorafenib. At the first diagnosis, the maximum section of the tumor was $15.0 \mathrm{~cm} \times 10.8 \mathrm{~cm}$, and the necrotic area was $39.47 \mathrm{~cm}^{2}$. After 4 cycles of treatment with Len combined with Nivolumab, the maximum section of the tumor was reduced to $13.0 \mathrm{~cm} \times 8.5 \mathrm{~cm}$, and the necrotic area was enlarged to $48.84 \mathrm{~cm}^{2}$. Finally, the enlarged right hepatic resection was performed successfully, and the patients were discharged safely and followed up regularly[36]. Won et al. reported a case of a BCLC C patient with double lesions on the right liver and tumor thrombus in the right anterior portal vein. AFP increased from $17,528 \mathrm{ng} / \mathrm{mL}$ to $30,070 \mathrm{ng} / \mathrm{mL}$ in just 12 days at admission. After 4 cycles Cobimetinib combined with Nivolumab treatment, the primary focus was reduced by $37.3 \%$, and the AFP was reduced by more than $99 \%$ from the treatment baseline (down to $12.9 \mathrm{ng} / \mathrm{mL}$ ). After the right hepatectomy, the histopathology revealed that a lesion was $100 \%$ necrotic, and The necrotic area of another was more than $80 \%$. There was active immune cell infiltration in the marginal tissue of the tumor. The patient was followed up every 3 months after operation, and there was no recurrence for 2 years[37]. Zhang et al. conducted a prospective study on the conversion therapy of advanced HCC with intrahepatic vascular tumor thrombus. In the treatment of 33 patients with Len combined with PD-1 inhibitors, the ORR was $45.5 \%$, the disease control rate(DCR) was $81.8 \%$, the conversion success rate based on imaging evaluation was $42.4 \%$, and the actual operation rate was $30.3 \%$, during the median follow-up time of 11.5 months, the recurrence-free rate was $60.0 \%$ at 6 months after surgery. As of November 2020, the median survival time and recurrence-free survival time had not yet reached the end point [38]. At the ASCO conference in 2020, Sun et al. reported that 60 patients who were unresectable in first diagnosis were treated with Len combined with PD-1 inhibitors, and 11 patients became resectable, and 9 of them had completed the operation. As of the last follow-up, 7 patients remained tumor-free [39]. In this patient, we used Len combined with Tor. After twice combined treatments, the patient's AFP dropped from baseline $>1210 \mathrm{ng} / \mathrm{ml}$ to $25.23 \mathrm{ng} / \mathrm{ml}$. The length, width and depth of the PVTT were significantly reduced, which provided the possibility of radical resection at the surgical technical level.

The indications for surgery after MTDs combined ICls therapy, the withdrawal time before operation, and whether to continue the combined therapy after surgery are still inconclusive. Our case did not benefit 
further in the two imaging evaluations of 2021.04.21 and 2021.05.06, and the classification of PVTT was reduced from $\otimes$ to $\otimes$. From the level of surgical technology, radical resection can be achieved. According to the recommendations of $\square$ Chinese expert consensus on Transforming Therapy of Advanced HCC based on Immunization combined with targeting regimen (2021 Edition) $\varangle[40] \varangle$ we performed " right hepatectomy plus cholecystectomy plus portal vein tumor thrombus removal and intestinal adhesion lysis" under general anesthesia on December 2021. The tumor-free results of postoperative pathology also suggested that the patient reached the standard of R0 resection. As an anti-angiogenic drug, Len may cause intraoperative and postoperative complications such as increased bleeding and delayed healing. The half-life of Len is about $28 \mathrm{~h}-35 \mathrm{~h}$, and the duration of interruption before invasive operation is at least 1 week [41]. We told the patient to discontinue the use of Len when the patient came to the hospital for review on May 5, 2021. As of the start of the operation, the patient stopped using Len for 7 days, stopped using Tor for 21 days. The intraoperative bleeding was $500 \mathrm{ml}$. The patient recovered well and was discharged smoothly 9 days after operation. No operation-related adverse events occurred. The patient was asked to review after one month. After evaluating the curative effect, we planned to continue treatment according to the same regimen for 6 to 12 months.

\section{Conclusion}

We report a case that advanced HCC with PVTT $₫$ was reduced to PVTT $\otimes$ after treatment with Len combined with Tor, and successfully completed conversion therapy to achieve R0 resection of the tumor. The good performance of MTDs combined with ICls has led to a new direction in the treatment of advanced HCC. Although there are many details to be further explored and studied in prospective randomized controlled trials, MTDs combined with ICls brings more radical opportunities for advanced patients.

\section{Abbreviations}

HCC: hepatocellular carcinoma; PVTT: portal vein tumor thrombus; PD-1: programmed cell death-1; PDL1: programmed cell death ligand-1; MTDs: molecular targeted drugs; TIM: the immune microenvironment of tumors; CT: computed tomography; MRI: magnetic resonance imaging; AFP: Alphafetoprotein.

\section{Declarations}

Acknowledgements

Not applicable. 


\section{Funding}

There was no funding for this article.

\section{Availability of data and materials}

The authors declare that all data and materials of the article are available to all readers of our article.

\section{Authors' contributions}

All authors contributed to the acquisition of data.JO performed the case analysis and drafted the manuscript; YZ, ZW conducts follow-up and data analysis; QL conducts analysis of surgery and pathological results; JZ participates in the formulation of preoperative treatment plans and operations. All authors read and approved the final manuscript.

\section{Ethics approval and consent to participate}

This study was performed in accordance with the principles of theDeclaration of Helsinki. The requirement for ethical approval was waived by the Henan Province Cancer Hospital Review Board.

\section{Consent for publication}

Written informed consent was obtained from the patient for the publication of this case report and the accompanying images.

\section{Competing interests}

All authors declare that they have no competing interests

\section{Author details}

${ }^{1}$ Department of Hepatobiliary and Pancreatic Surgery, Henan Cancer Hospital, 127 DongMing St., Jinshui Dis., Zhengzhou, Henan 450003, China.

\section{References}

1. Villanueva A. Hepatocellular Carcinoma. N Engl J Med. 2019;380:1450-62.

2. Llovet JM, Ricci S, Mazzaferro V, Hilgard P, Gane E, Blanc JF, et al. Sorafenib in advanced hepatocellular carcinoma. N Engl J Med. 2008;359:378-90.

3. Kudo M, Finn RS, Qin S, Han KH, Ikeda K, Piscaglia F, et al. Lenvatinib versus sorafenib in first-line treatment of patients with unresectable hepatocellular carcinoma: a randomised phase 3 noninferiority trial. Lancet. 2018;391:1163-73.

4. Sangro B, Sarobe P, Hervás-Stubbs S, Melero I. Advances in immunotherapy for hepatocellular carcinoma. Nat Rev Gastroenterol Hepatol. 2021;18:525-43. 
5. Susan J. Keam. Toripalimab: First Global Approval.Drugs.2019;79:573 - 78.

6. Li J, Kan H, Zhao L, Sun Z, Bai C. Immune checkpoint inhibitors in advanced or metastatic mucosal melanoma: a systematic review. Ther Adv Med Oncol. 2020;12:1758835920922028.

7. Fukumura D, Kloepper J, Amoozgar Z, Duda DG, Jain RK. Enhancing cancer immunotherapy using antiangiogenics: opportunities and challenges. Nat Rev Clin Oncol. 2018;15:325-40.

8. He MK, Liang RB, Zhao Y, Xu YJ, Chen HW, Zhou YM, et al. Lenvatinib, toripalimab, plus hepatic arterial infusion chemotherapy versus lenvatinib alone for advanced hepatocellular carcinoma. Ther Adv Med Oncol. 2021;13:17588359211002720.

9. Llovet JM, De Baere T, Kulik L, Haber PK, Greten TF, Meyer T, et al. Locoregional therapies in the era of molecular and immune treatments for hepatocellular carcinoma. Nat Rev Gastroenterol Hepatol. 2021;18:293-313.

10. Sitzmann JV, Abrams R. Improved survival for hepatocellular cancer with combination surgery and multimodality treatment. Ann Surg. 1993;217:149-54.

11. Llovet JM, Kelley RK, Villanueva A, Singal AG, Pikarsky E, Roayaie S, et al. Hepatocellular carcinoma. Nat Rev Dis Primers. 2021;7:6.

12. Folprecht G, Gruenberger T, Bechstein WO, Raab HR, Lordick F, Hartmann JT, et al. Tumour response and secondary resectability of colorectal liver metastases following neoadjuvant chemotherapy with cetuximab: the CELIM randomised phase 2 trial. Lancet Oncol. 2010;11:38-47.

13. Sung H, Ferlay J, Siegel RL, Laversanne M, Soerjomataram I, Jemal A, et al. Global Cancer Statistics 2020: GLOBOCAN Estimates of Incidence and Mortality Worldwide for 36 Cancers in 185 Countries. CA Cancer J Clin. 2021;71:209-49.

14. Jemal A, Ward EM, Johnson CJ, Cronin KA, Ma J, Ryerson B, et al. Annual Report to the Nation on the Status of Cancer, 1975-2014, Featuring Survival. J Natl Cancer Inst. 2017;109.

15. Lu J, Zhang XP, Zhong BY, Lau WY, Madoff DC, Davidson JC, et al. Management of patients with hepatocellular carcinoma and portal vein tumour thrombosis: comparing east and west. Lancet Gastroenterol Hepatol. 2019;4:721-30.

16. Zhang ZM, Lai EC, Zhang C, Yu HW, Liu Z, Wan BJ, et al. The strategies for treating primary hepatocellular carcinoma with portal vein tumor thrombus. Int J Surg. 2015;20:8-16.

17. Ikai I, Yamamoto Y, Yamamoto N, Terajima H, Hatano E, Shimahara Y, et al. Results of hepatic resection for hepatocellular carcinoma invading major portal and/or hepatic veins. Surg Oncol Clin $\mathrm{N}$ Am. 2003;12:65-75. ix.

18. Shuqun C, Mengchao W, Han C, Feng S, Jiahe Y, Guanghui D, et al. Tumor thrombus types influence the prognosis of hepatocellular carcinoma with the tumor thrombi in the portal vein. Hepatogastroenterology. 2007;54:499-502.

19. EASL Clinical Practice Guidelines. Management of hepatocellular carcinoma. J Hepatol. 2018;69:182-236. 
20. Benson AB, D39, Angelica MI, Abbott DE, Anaya DA, Anders R, et al. Hepatobiliary Cancers, Version 2.2021, NCCN Clinical Practice Guidelines in Oncology. J Natl Compr Canc Netw. 2021;19:541 - 65.

21. Specifications for the diagnosis. and treatment of primary liver cancer (2019 edition). Chin $\mathrm{J}$ Hepatol. 2020;28:112-28.

22. Peng ZW, Guo RP, Zhang YJ, Lin XJ, Chen MS, Lau WY. Hepatic resection versus transcatheter arterial chemoembolization for the treatment of hepatocellular carcinoma with portal vein tumor thrombus. Cancer. 2012;118:4725-36.

23. Liang L, Chen TH, Li C, Xing H, Han J, Wang MD, et al. A systematic review comparing outcomes of surgical resection and non-surgical treatments for patients with hepatocellular carcinoma and portal vein tumor thrombus. HPB (Oxford). 2018;20:1119-29.

24. El-Khoueiry AB, Sangro B, Yau T, Crocenzi TS, Kudo M, Hsu C, et al. Nivolumab in patients with advanced hepatocellular carcinoma (CheckMate 040): an open-label, non-comparative, phase 1/2 dose escalation and expansion trial. Lancet. 2017;389:2492-502.

25. Zhu AX, Finn RS, Edeline J, Cattan S, Ogasawara S, Palmer D, et al. Pembrolizumab in patients with advanced hepatocellular carcinoma previously treated with sorafenib (KEYNOTE-224): a nonrandomised, open-label phase 2 trial. Lancet Oncol. 2018;19:940-52.

26. Qin S, Ren Z, Meng Z, Chen Z, Chai X, Xiong J, et al. Camrelizumab in patients with previously treated advanced hepatocellular carcinoma: a multicentre, open-label, parallel-group, randomised, phase 2 trial. Lancet Oncol. 2020;21:571-80.

27. Finn RS, Qin S, Ikeda M, Galle PR, Ducreux M, Kim TY, et al. Atezolizumab plus Bevacizumab in Unresectable Hepatocellular Carcinoma. N Engl J Med. 2020;382:1894-905.

28. Finn RS, Ikeda M, Zhu AX, Sung MW, Baron AD, Kudo M, et al. Phase Ib Study of Lenvatinib Plus Pembrolizumab in Patients With Unresectable Hepatocellular Carcinoma. J Clin Oncol. 2020;38:2960-70.

29. Xu J, Zhang Y, Jia R, Yue C, Chang L, Liu R, et al. Anti-PD-1 Antibody SHR-1210 Combined with Apatinib for Advanced Hepatocellular Carcinoma, Gastric, or Esophagogastric Junction Cancer: An Open-label, Dose Escalation and Expansion Study. Clin Cancer Res. 2019;25:515-23.

30. Yau Thomas KY, Koo K, Tae-You A, Santoro AB, El-Khoueiry, et al. Nivolumab (NIVO) + ipilimumab (IPI) combination therapy in patients (pts) with advanced hepatocellular carcinoma (aHCC): Results from CheckMate 040. J Clin Oncol. 2019;37 Suppl :4012.

31. Kelley Robin S, Bruno H, William T, Okusaka M, Ikeda, et al. Efficacy, tolerability, and biologic activity of a novel regimen of tremelimumab (T) in combination with durvalumab (D) for patients (pts) with advanced hepatocellular carcinoma (aHCC). Journal of Clinical Oncology 2020;38 Suppl:4508.

32. He M, Li Q, Zou R, Shen J, Fang W, Tan G, et al. Sorafenib Plus Hepatic Arterial Infusion of Oxaliplatin, Fluorouracil, and Leucovorin vs Sorafenib Alone for Hepatocellular Carcinoma With Portal Vein Invasion: A Randomized Clinical Trial. JAMA Oncol. 2019;5:953-60.

33. Choi C, Choi GH, Kim TH, Tanaka M, Meng MB, Seong J. Multimodality Management for Barcelona Clinic Liver Cancer Stage C Hepatocellular Carcinoma. Liver Cancer. 2014;3:405-16. 
34. Makary MS, Khandpur U, Cloyd JM, Mumtaz K, Dowell JD. Locoregional Therapy Approaches for Hepatocellular Carcinoma: Recent Advances and Management Strategies. Cancers (Basel). 2020;12.

35. He MK, Le Y, Li QJ, Yu ZS, Li SH, Wei W, et al. Hepatic artery infusion chemotherapy using mFOLFOX versus transarterial chemoembolization for massive unresectable hepatocellular carcinoma: a prospective non-randomized study. Chin J Cancer. 2017;36:83.

36. Chen $X$, Zhang Y, Zhang N, Ge Y, Jia W. Lenvatinib combined nivolumab injection followed by extended right hepatectomy is a feasible treatment for patients with massive hepatocellular carcinoma: a case report. Onco Targets Ther. 2019;12:7355-9.

37. Ho WJ, Sharma G, Zhu Q, Stein-O\&\#39, Brien G, Durham J, et al. Integrated immunological analysis of a successful conversion of locally advanced hepatocellular carcinoma to resectability with neoadjuvant therapy. J Immunother Cancer. 2020;8.

38. Zhang W, Hu B, Han J, Wang H, Wang Z, Chen M, et al. A preliminary report on the study of the combination of PD-1 inhibitors and multi-target tyrosine kinase inhibitors for the conversion therapy of advanced hepatocellular carcinoma. Chin J Hepatol.2020;26:947-8.

39. Sun HC, Zhu XD, Huang C, Shen YH, Ji Y et al. Initially unresectable hepatocellular carcinoma treated by combination therapy of tyrosine kinase inhibitor and anti-PD-1 antibody followed by resection. $J$ Clin Oncol 2020;38 Suppl『e16690.

40. Chinese Expert Consensus on Transformation Therapy of Advanced Hepatocellular Carcinoma Based on Immune Combination Targeting Program (2021 Edition). Chin J Hepatol.2021;27:241-51.

41. Dubbelman AC, Rosing $H$, Nijenhuis $C$, Huitema AD, Mergui-Roelvink $M$, Gupta $A$, et al. Pharmacokinetics and excretion of (14)C-lenvatinib in patients with advanced solid tumors or lymphomas. Invest New Drugs. 2015;33:233-40.

\section{Figures}




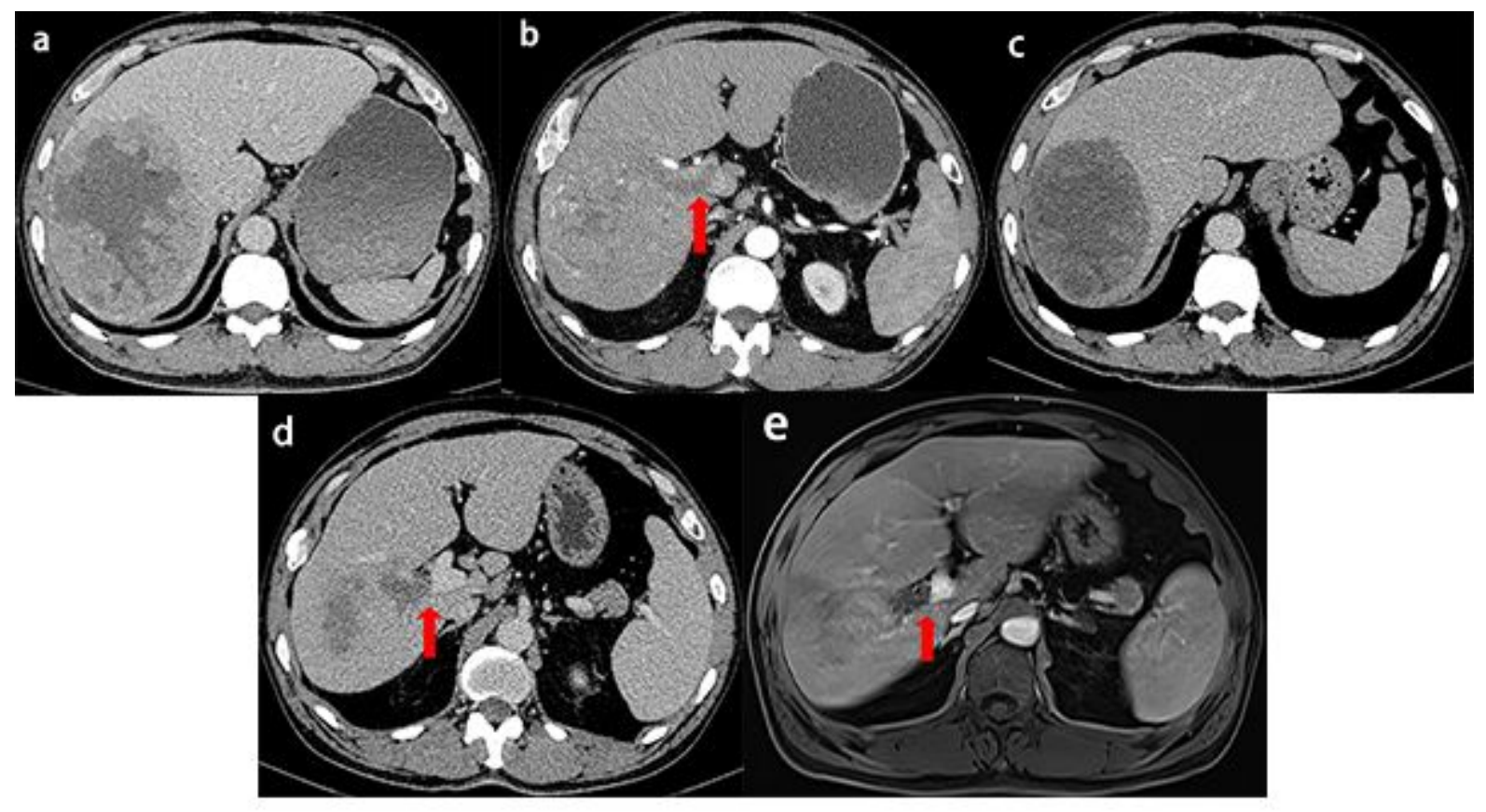

\section{Figure 1}

Comparison of enhanced CT and enhanced MRI before and after combined treatment: (a) no-enhanced CT on Feb.19,2021: a huge mass in the right lobe of the liver with large area necrosis; (b) Arterial phase of enhanced CT on Feb.19,2021: uneven enhancement of the mass, tumor thrombus in the right branch and the main stem of the portal vein (arrow); (c) no-enhanced CT on April 22,2021: the mass of the right lobe of the liver was smaller than before, and the necrotic area was enlarged; (d) Delayed phase of enhanced CT on April 22,2021: the PVTT shrunk and was confined to the right branch of the portal vein; (e) Enhanced MRI on May 6, 2021: the PVTT was confined to the right branch. 


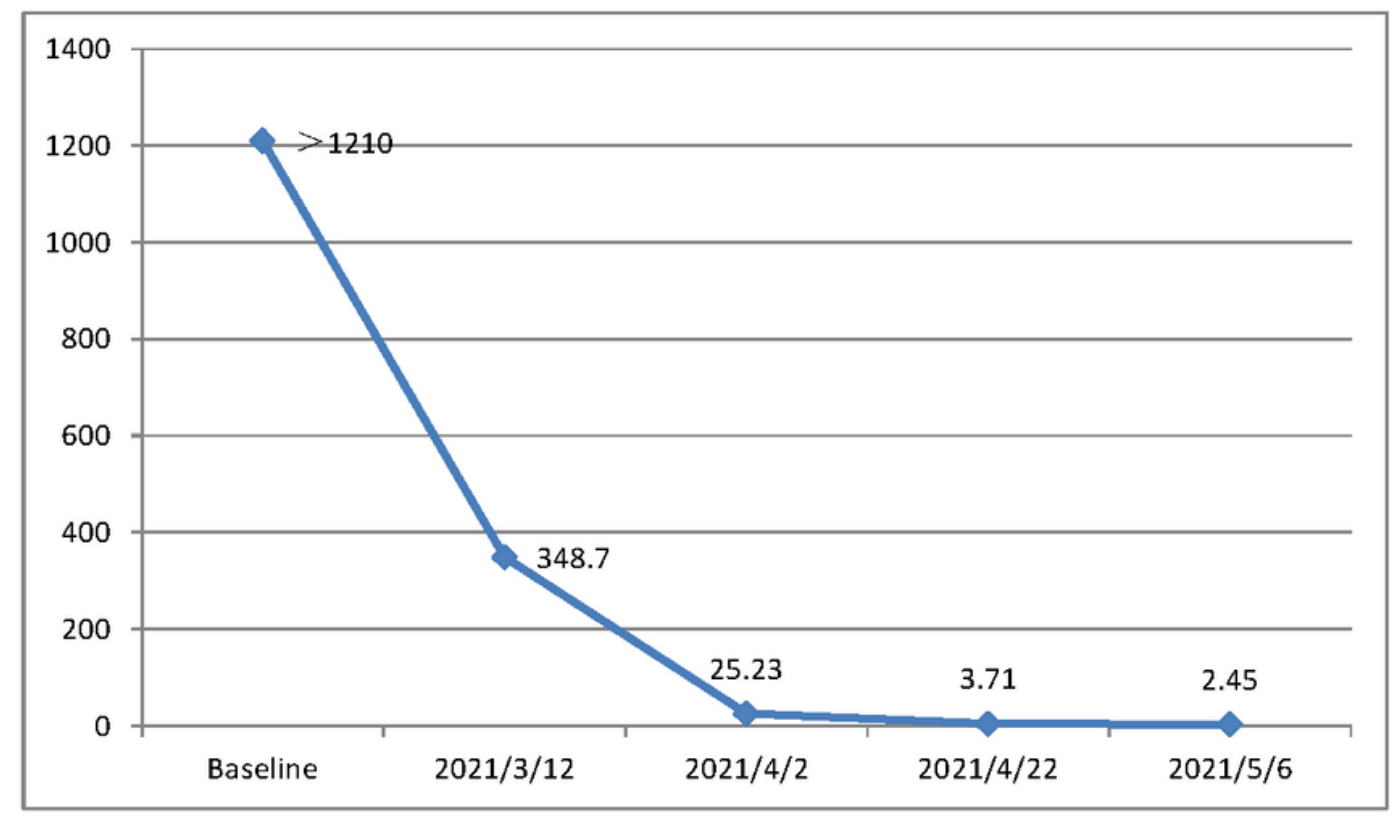

Figure 2

Line chart of changes in AFP during preoperative neoadjuvant therapy $(\mathrm{ng} / \mathrm{ml})$
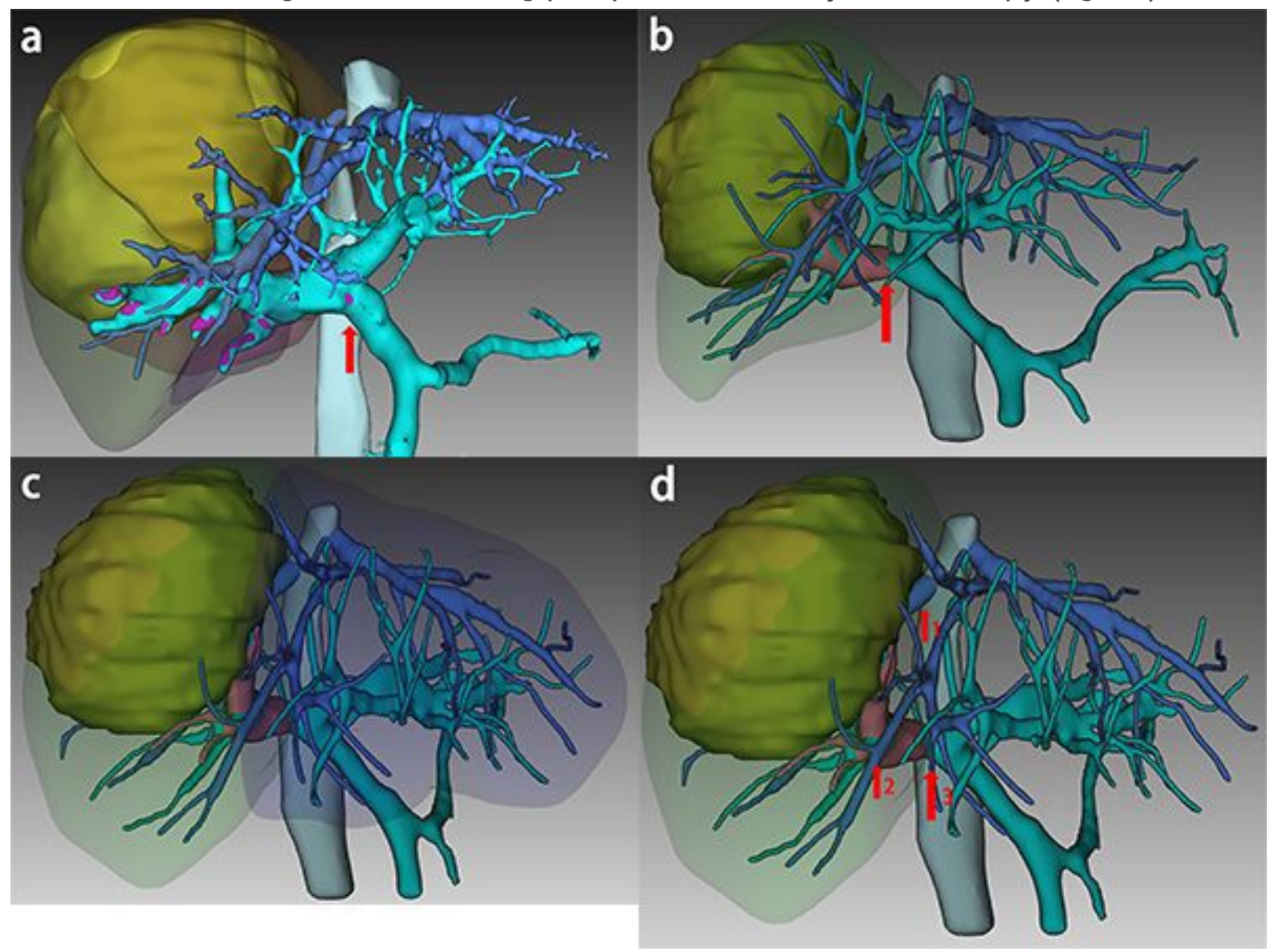

Page $16 / 17$ 


\section{Figure 3}

The 3D visualization results based on the CT image on February 19, 2021 and the MRI image on May 6, 2021区 (a) tumor thrombus invaded the main portal vein before neoadjuvant therapy; (b) tumor thrombus shrunk and confined to the right branch of the portal vein after neoadjuvant therapy; (c) the extent of resection simulated by the proposed right hepatectomy (d) right hepatectomy mainly cut off the right hepatic vein (arrows 1), the right half hepatic branch of the middle hepatic vein (arrows 2 ) and the right branch of the portal vein (arrows 3).

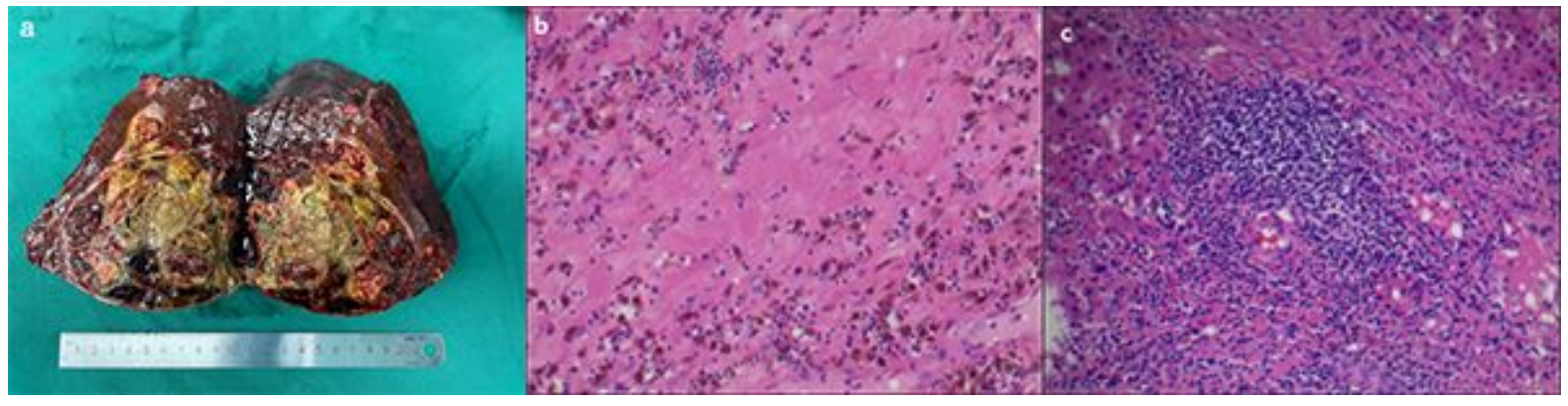

\section{Figure 4}

Gross specimens and histopathological results: (a)Specimen of the right liver; (b)Histopathology of the mass showed hyperplasia of fibrous tissue, infiltration of inflammatory cells and hemosiderin; (c)Tumor thrombus showed degenerative necrotic substance and a large number of inflammatory cells infiltration in a few of fibrous connective tissue. 